\title{
Building Green Cloud Services at Low Cost
}

\author{
Josep Ll. Berral ${ }^{\dagger \ddagger}$, Íñigo Goiri, Thu D. Nguyen, Ricard Gavaldà ${ }^{\dagger}$, Jordi Torres ${ }^{\dagger \ddagger}$, Ricardo Bianchini \\ $\ddagger$ Computer Architecture Department, ${ }^{\dagger}$ Department of Software \\ Universitat Politecnica de Catalunya, Barcelona Supercomputing Center \\ Barcelona, Spain \\ \{berral,torres\}@ac.upc.edu, gavalda@lsi.upc.edu \\ Department of Computer Science \\ Rutgers University \\ Piscataway, USA \\ $\{$ goiri,tdnguyen,ricardob\}@cs.rutgers.edu
}

\begin{abstract}
Interest in powering datacenters at least partially using on-site renewable sources, e.g. solar or wind, has been growing. In fact, researchers have studied distributed services comprising networks of such "green" datacenters, and load distribution approaches that "follow the renewables" to maximize their use. However, prior works have not considered where to site such a network for efficient production of renewable energy, while minimizing both datacenter and renewable plant building costs. Moreover, researchers have not built real load management systems for follow-the-renewables services. Thus, in this paper, we propose a framework, optimization problem, and solution approach for siting and provisioning green datacenters for a follow-the-renewables HPC cloud service. We illustrate the location selection tradeoffs by quantifying the minimum cost of achieving different amounts of renewable energy. Finally, we design and implement a system capable of migrating virtual machines across the green datacenters to follow the renewables. Among other interesting results, we demonstrate that one can build green HPC cloud services at a relatively low additional cost compared to existing services.
\end{abstract}

Keywords-datacenter; renewable energy; green computing

\section{INTRODUCTION}

Motivation. It is well known that datacenters are massive consumers of electricity. This consumption leads to high carbon emissions, since most of this electricity is produced using fossil fuels. Increasing awareness of these emissions is driving demand for cleaner products and services. Thus, many companies are starting to build "green" datacenters, i.e. datacenters with on-site renewable power plants. For example, Apple [1] and McGraw-Hill [2] have built 20MW and 14MW solar arrays, respectively, for their datacenters. A few small cloud providers [3]-[5] operate datacenters powered completely by on-site solar and/or wind farms. As carbon taxes and cap-and-trade start to migrate from Europe and Asia to the US, even more companies are likely to build green datacenters. For example, the UK government created a mandatory cap-and-trade scheme for businesses consuming more than $6 \mathrm{GWh}$ per year [6]; i.e. a company with even a small $700-\mathrm{kW}$ datacenter must participate.

Some research efforts have explored how to incorporate sources of renewable ("green") energy such as solar and wind into datacenters. In particular, several studies have considered load distribution between geographically distributed datacenters to take advantage of green energy produced onsite [7]-[10]. Two key observations behind these works are: (1) services are often replicated on multiple geographically distributed datacenters for high availability and low response time, and (2) diverse generation of green energy because of differing local weather conditions. Thus, load distribution approaches that consider green energy production can allow the workload to "follow the renewables" to increase green energy usage. However, to the best of our knowledge, no previous work has considered where to site such a network of datacenters globally for efficient production of green energy, while minimizing both datacenter and renewable plant building costs. Moreover, prior works have not built real load management systems for follow-the-renewables cloud services. These are exactly the topics of this paper.

Selecting sites. A service provider seeking to create a network of green datacenters must consider the significant cost of building, provisioning, and operating these datacenters. This cost depends heavily on the locations of the datacenters. For example, the production of green energy from sources such as solar and wind depends strongly on location.

Thus, in this paper, we study the siting and provisioning of a network of green datacenters to provide a given level of computing power (e.g., $50 \mathrm{MW}$ ) for a follow-the-renewables high-performance computing (HPC) cloud service, where a desired fraction of the energy must be produced by on-site green power plants (the rest coming from the electrical grid).

We first propose a framework for selecting locations and provisioning the datacenters and green power plants at these locations. The framework includes parameters representing all aspects of datacenter costs, workload migration to follow the renewables, and availability. (As we target batch HPC workloads, the network latency to clients is not a relevant issue.) The framework allows us to define the site selection and provisioning as a Mixed Integer Linear Programming (MILP) problem. We also propose a heuristic-based approach that turns the MILP optimization into a set of linear problems to make it solvable for a large set of locations.

Based on our framework, optimization approach, and real location data, we built a tool for selecting locations and 
provisioning the datacenters. The tool is extensible so that new parameters and constraints can be added. We use the tool to explore the cost of different desired percentages of green energy, using different approaches for energy storage, using solar and/or wind sources, and the overhead of load migration. Our results show that green cloud services can be built at relatively low cost, e.g. 13\% when the provider desires $50 \%$ green energy, compared to existing services.

Managing workloads. Finally, a follow-the-renewables HPC cloud service needs to intelligently migrate virtual machines (VMs) based on the availability of green energy at the datacenters. However, we are not aware of any real VM management systems for follow-the-renewables cloud services, since the prior work in this area has focused either on simulations or modeling [8]-[12]. (Le et al. [7] built a prototype request distribution system for an Internet service, not a VM management system.) To illustrate the feasibility of building such a system, we design and implement GreenNebula, an extension of the OpenNebula within-datacenter VM management system [13]. GreenNebula uses live VM migration and a distributed file system across the datacenters. Using emulation, we demonstrate that GreenNebula can migrate live VMs to follow renewables with low overheads.

Contributions: (1) We propose a framework, optimization problem, and solution approach for siting and provisioning green datacenters for a follow-the-renewables HPC cloud service; and (2) We design and implement a system for lowoverhead live VM migration in such a service.

Other approaches for greening cloud services. While our work considers on-site green energy generation, we do not argue that this is the best approach for all operators. For example, Google prefers to invest in renewables by financing new plants and pumping the produced energy into the electrical grid. Rather, we argue that on-site generation will be the approach of choice for many operators, as suggested by [1]-[4], [14], regardless of whether this choice is made for marketing positioning, public relations, or environmental reasons. Moreover, despite their decreasing-butstill-high capital costs, exploiting solar and/or wind energy in datacenters may reduce energy costs [15], peak grid power cost [16], or both [17], [18]. We expect that an increasing number of companies will benefit from on-site renewables.

\section{Placement FRAMEWORK}

We seek to efficiently select a set of locations, and provision datacenters at these locations to support a given constant amount of compute power. Each datacenter will have an on-site green power plant (either solar, wind, or both) so that together, a desired percentage of energy used by the datacenters will come from green sources.

\section{A. Framework parameters}

Table I lists the entire set of parameters in our framework. They range from inputs given by the cloud service provider to parameters that we seek to instantiate via optimization. Among the more interesting parameters are those related to costs, green energy generation, and availability.

Costs. The overall cost of a network of datacenters can be broken down into capital (CAPEX) and operational (OPEX) components. The CAPEX costs are those investments made upfront and depreciated over the lifetime of the datacenters. CAPEX can be further divided into capital costs that are independent of ( $C A P \_$ind $)$and those that are dependent on ( $C A P \_$dep $)$the number of servers to be hosted.

The $C A P \_$ind $(d)$ costs for a datacenter at a location $d$ relate to bringing grid or "brown" electricity (costLinePow) and external networking (costLineNet) to the datacenter. (Although the amount of grid electricity and external bandwidth depends on the number of servers, the base cost of laying out any transmission line and/or optical fiber dominates.) These costs can be estimated from the distance between the location and (1) the closest transmission line or power plant; and (2) the closest network backbone.

The $C A P \_d e p(d)$ costs relate to land acquisition $($ landCost $(d))$, construction of the datacenter and green power plants (buildCost $(d)$ ), including the purchase and installation of the power delivery, backup, and cooling infrastructures, the purchase of extra batteries for storing green energy (battCost $(d))$, purchasing servers (serverCost $(d)$ ) and (internal) networking equipment (switchCost $(d)$ ) to be hosted by the datacenters. (Besides using batteries, our framework considers storing green energy into the electrical grid for later use; this approach is typically called "net metering".) The land price varies according to location, whereas the other costs do not to a first approximation. Most of these costs depend on the level of redundancy that will be built into each datacenter. The construction cost is typically estimated as a function of the maximum power to be consumed by the datacenter. This maximum power is that required by the maximum number of servers (and networking gear) when running at $100 \%$ utilization times the maximum expected PUE of the datacenter. The PUE is computed by dividing the overall power consumption by the power consumption of the computational equipment. The PUE is higher when temperature and/or humidity are high, since cooling consumes more energy under those conditions.

The OPEX costs (OP) are those incurred during the operation of the datacenters, and include costs for external network bandwidth use and the brown electricity required to run the datacenters. (There is also a cost for water, which is currently not considered but can be easily added.) The brown electricity cost can be computed based on the IT equipment's energy consumption, the PUE, the amount of green energy generated on-site, and the brown electricity price. Both the brown electricity price and the PUE vary with location.

Lower taxes and one-time incentives are another important cost component. For example, some states in the US lower taxes on datacenters. This component depends on the nature 
TABLE I. FRAMEWORK PARAMETERS. EACH LOCATION $d$ BELONGS TO THE SET $D$. EACH $t$ IS A FIXED TIME PERIOD WITHIN A LONGER DURATION $T$.

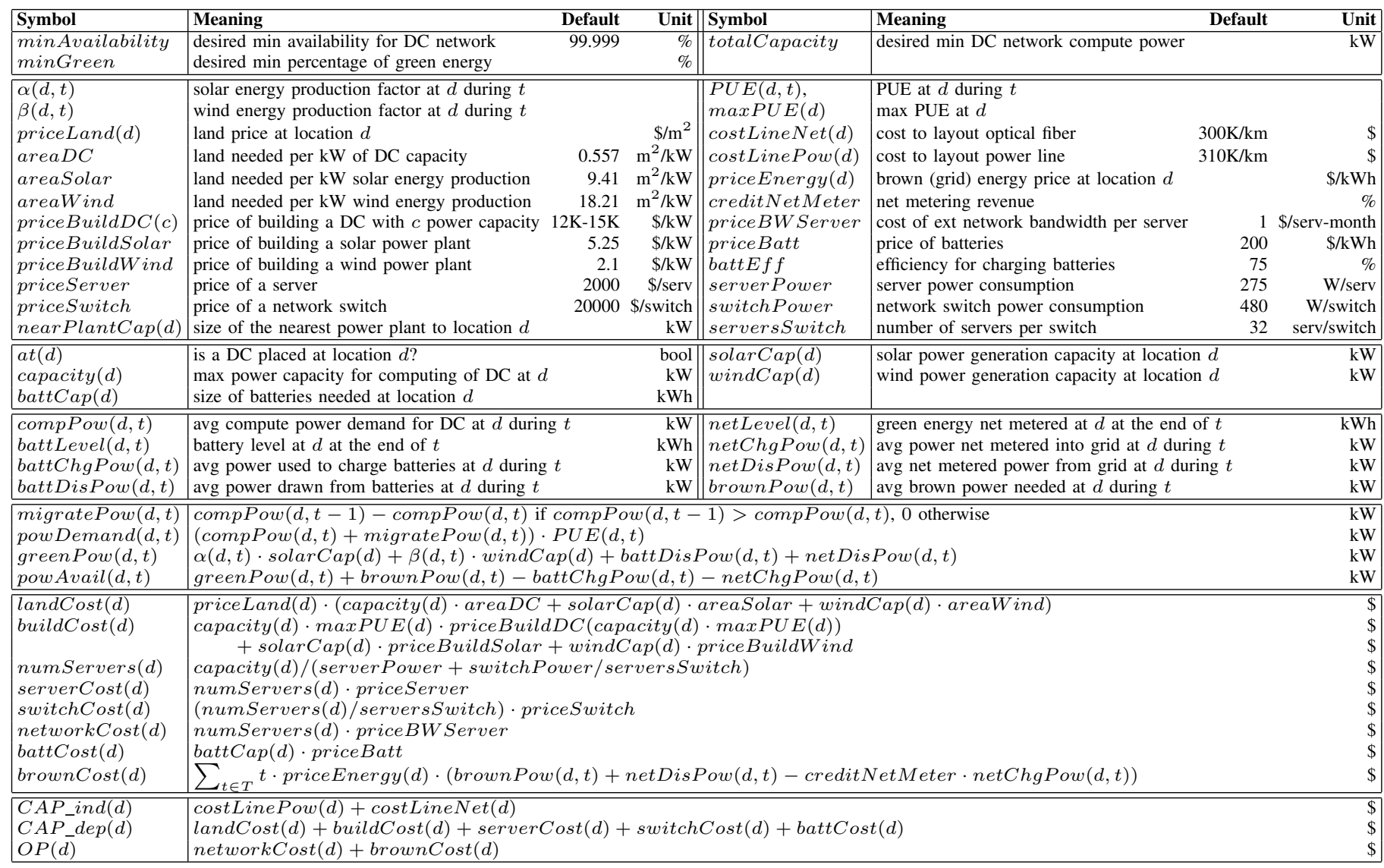

of the savings and applies to other costs in different ways. Although we do not consider this component further, it is easy to add it to our framework.

Finally, as we have already noted, network latency to clients is not a relevant issue since we are targeting HPC workloads. If network latency is an important consideration, it can be added to our optimization framework as in [19]. Similarly, the availability of a local IT workforce for maintenance of the datacenter can be an important consideration that we do not currently model; it can be incorporated as either a cost, or a placement constraint.

Green energy generation. Two key factors that affect the cost and benefit of building green power plants include efficiency and capacity factor. Efficiency refers to the percentage of sunlight energy and wind energy that is transformed into DC electricity by solar and wind energy plants, respectively. The efficiency of today's most affordable PV technology (multi-crystalline silicon) hovers around $15 \%$. The efficiency of today's wind turbines is close to $50 \%$. There are also losses as the generated DC power is converted to AC to supply the datacenter. In our framework, we combine the efficiency and conversion losses of power generation in the functions $\alpha(d, t)$ and $\beta(d, t)$, which are the percentage of the installed capacity a solar and wind farm, respectively, would generate at location $d$ in time $t$.

Capacity factor refers to the percentage of the maximum theoretical energy production (e.g., 24 hours of maximum sunlight every day for a solar power plant) that is actually produced. Capacity factors vary depending on location and weather. For example, Berlin (Germany), New York (US), Canberra (Australia), and Phoenix (US) have solar capacity factors of approximately $13.5 \%, 16.4 \%, 20.2 \%$, and $22.9 \%$, and wind capacity factors of approximately $3.4 \%, 18.9 \%$, $8.4 \%$, and $3.4 \%$, respectively. ${ }^{1}$ In our framework, the solar and wind capacity factors are aggregations of $\alpha(d, t)$ and $\beta(d, t)$, respectively, over a time period (e.g., a year).

Datacenter network availability. We model availability as $\sum_{i=0}^{n-1}\left(\begin{array}{c}n \\ i\end{array}\right) \cdot a^{n-i} \cdot(1-a)^{i}$, where $n$ is the number of datacenters and $a$ is the availability of each datacenter. This model computes the probability that at least one datacenter will be available when the service is needed, and has been used in multiple prior works, e.g. [19], [20]. The availability of each datacenter depends on its redundancy level. At one extreme, Tier I datacenters have a single path for power and cooling distribution. At the other extreme, Tier IV datacenters have two active power and cooling paths, with redundant components in each path. Existing Tier I

\footnotetext{
${ }^{1}$ http://apps1.eere.energy.gov/buildings/energyplus/weatherdata_about.cfm
} 


$$
\text { TotalCost }=\sum_{d \in D} a t(d) \cdot\left(C A P \_i n d(d)+C A P \_d e p(d)+O P(d)\right)
$$

1. $\forall d, \forall t: \operatorname{capacity}(d) \geq$ migratePow $(d, t)+\operatorname{computePow}(d, t)$

2. $\forall t: \sum_{d}$ computePow $(d, t) \geq$ totalCapacity

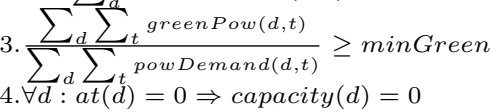

5. $\forall d, \forall t:$ powDemand $(d, t)<\operatorname{powAvail}(d, t)$

6. $\forall d, \forall t:$ battLevel $(d, t)=$ battLevel $(d, t-1)+$

battEff $\cdot$ battChgPow $(d, t) \cdot t-\operatorname{battDisPow}(d, t) \cdot t$

7. $\forall d, \forall t: 0<\operatorname{battLevel}(d, t)<\operatorname{battCap}(d)$

8. $\forall d, \forall t:$ net Level $(d, t)=$ netLevel $(d, t-1)+\operatorname{netChgPow}(d, t) \cdot t-$ netDisPow $(d, t) \cdot t$

9. $\forall d, \forall t:$ netLevel $(d, t) \geq 0$

10. $\forall d, \forall t$ : brownPow $(d, t)<$ nearPlantCap $(d) \cdot F$

11.Availability $\geq$ minAvailability

Figure 1. Optimization problem. $F$ is a percentage cap on the maximum power that the datacenter can draw from the nearest brown plant.

datacenters achieve an availability of $99.67 \%$, whereas Tier II achieves $99.74 \%$, Tier III achieves $99.98 \%$, and Tier IV achieves $99.995 \%$ [21].

\section{B. Formulating the optimization problem}

The HPC cloud service provider seeks to select locations from a set of potential locations $(D)$ for a datacenter network with a given compute power (e.g., 25MW), and a desired percentage (e.g., 80\%) of the energy coming from on-site green sources. The optimization goal is to minimize the overall cost of the datacenter network, while respecting the power, percentage of green energy, and availability constraints. The inputs to the optimization are (1) the minimum total power capacity for computing that must be supported by the network at any given point in time, (2) the tier of the datacenters and the overall minimum availability, (3) the minimum percentage of green energy, (4) the CAPEX and OPEX costs for each location, and (5) the sizes of the power plants that can be used to supply the datacenters with brown energy (since this constrains the maximum datacenter size that can be placed at a given location). The outputs are the lowest cost found, the locations, and, at each location, the datacenter power capacity, the green-energy generation capacity, and the battery storage capacity (if any).

Equation 1 in Fig. 1 defines the cost we seek to minimize (TotalCost), where at $(d)$ is a boolean representing the placement of a datacenter at location $d$. Recall that $C A P \_i n d(d)$ is the CAPEX cost of the datacenter at $d$ that is independent of datacenter size, $C A P_{-} \operatorname{dep}(d)$ is the CAPEX cost that depends on size, and $O P$ is the OPEX cost. As the production of green energy varies over time, the optimization divides time into discrete slots and computes the power drawn from different sources (powAvail) for each slot. It also computes the amount of energy stored and drawn from batteries and/or the grid (via net metering) for each slot.

We pessimistically assume that load must be migrated between datacenters when following the renewables; in practice, it may be possible to direct arriving jobs to the right datacenter, rather than migrating running jobs. Jobs continue to execute during migrations; such live migration is currently supported in many virtualized environments, including OpenNebula, which is the basis for our GreenNebula implementation (Section V). When load is migrated from one datacenter to the next, we assume that it consumes energy at both sites for the entire next epoch (migratePow $(d, t)$ ). That is, if $1 \mathrm{MW}$ of load is migrated from datacenter $d_{1}$ to $d_{2}$ at the end of epoch $t-1$, then we assume that the $1 \mathrm{MW}$ is consumed at both $d_{1}$ and $d_{2}$ during epoch $t$. In Section IV-E, we study the sensitivity of our results to this assumption.

TotalCost should be minimized under the constraints in Fig. 1. The constraints include power consumption and generation capacity, minimum percentage of green energy, battery capacity [18], green energy storage, brown power demand, and availability. The general formulation allows green energy to be stored in batteries (battLevel $(d, t)$ and/or the grid via net metering (netLevel $(d, t)$ ). In some evaluation studies below, we assume net metering and count the net metered green energy toward the green energy requirement. In scenarios where net metering is disallowed, we simply constrain netLevel $(d, t)$ to always be 0 . In scenarios without batteries, we constrain battCap $(d)$ to be 0 .

While not shown for simplicity, all CAPEX costs are assumed to be financed at a given interest rate. Further, we assume that land costs are fully recoverable; that is, land can be sold at the end of the datacenter lifetime for at least the purchasing price. Thus, the only land cost is that of financing the purchase, rather than the purchasing cost itself. Finally, the availability constraint we use is more complex than in the figure. Specifically, in a network with $n$ datacenters and $S$ servers, we also ensure that the failure of $n-1$ datacenters will leave $S / n$ servers available to handle the load.

\section{Heuristic solver}

The above optimization problem can be formulated as a MILP problem and solved using a MILP solver. However, some of the constraints make it time-consuming to solve, especially since we consider costs over an entire year to account for the full range of weather conditions. Thus, we develop a heuristic-based optimization approach.

Our approach has three parts: (1) filter out the locations that are unlikely to be in the solution to reduce the size of $D$, (2) transform the MILP problem into an LP problem by specifying the siting (i.e., providing $a t(d)$ as an input) and whether each datacenter should be small or large, and (3) use a procedure similar to simulated annealing (SA) to search for a good solution.

To filter out locations, we calculate the cost of each location for some common configurations (e.g., solar-only, a combination of solar and wind). Then, we remove locations with high costs and subsets of locations that are similar (e.g., same time zone, similar costs and PUEs). In our evaluations with 1373 locations, we typically remove all 
but 50-100 locations. We have also considered less filtering, e.g. leaving 200 locations, but did not find significant improvements in the solutions.

We then start the SA search by siting a small datacenter at each of the unfiltered locations, and solve the resulting LP problem for the provisioning of the datacenters and the corresponding costs. Next, we generate neighboring solutions for the search by: (1) removing a datacenter (i.e., set at $(d)$ to 0 for some $d$ ), (2) adding a datacenter, (3) changing the size of a datacenter, and/or (4) merging two datacenters into a larger one. We run the search until a threshold of consecutive iterations do not improve the solution.

We parallelize our search by running multiple instances of it with different neighbor generation approaches on multiple cores. Each instance follows a different search path, and they periodically synchronize to get the current best solution.

\section{Placement Tool And Input Data}

We created a tool based on our framework and optimization machinery. It takes as input the desired computing power, the minimum percentage of green energy, and the minimum availability. The tool outputs the number of datacenters, their locations, their provisioning including onsite green power plants, and costs. Next, we instantiate the parameters in Table I. We then quantify the cost of building datacenters at different locations. Finally, we detail the results of a case study.

\section{A. Instantiating the framework parameters}

Meteorological data. We obtained Typical Meteorological Year (TMY) information for 1373 unique locations worldwide from the US Department of Energy. ${ }^{2}$ A TMY is a 1-year dataset of hourly weather values selected to include a representative range of weather phenomena for a location, while still giving annual averages that are consistent with the long-term averages for the location. The TMY for each location includes temperature, solar irradiation, air pressure, and wind speed. We use this set of 1373 locations (Fig. 2) as the set $D$ of possible locations for siting datacenters.

The top map in Fig. 2 plots the 1373 locations. Observe from the middle and bottom maps in Fig. 2 that, while our set of locations is most dense for North America, Europe, and parts of Asia, there is good coverage of most world areas with good wind and solar availability.

Solar and wind capacity factors. We compute the average solar power generated using $\alpha(d, t)$, which we derive using TMY solar irradiation and temperature data, an efficiency of $15 \%$, and conversion losses [23]. The solar capacity factor of a location $d$ is then the average of its $\alpha(d, t)$, over all $t$, in a year. Similarly, we compute the average wind power generated using $\beta(d, t)$, which we derive using the

\footnotetext{
${ }^{2}$ http://apps1.eere.energy.gov/buildings/energyplus/weatherdata_about. $\mathrm{cfm}$. We removed duplicates and locations with incomplete data.
}

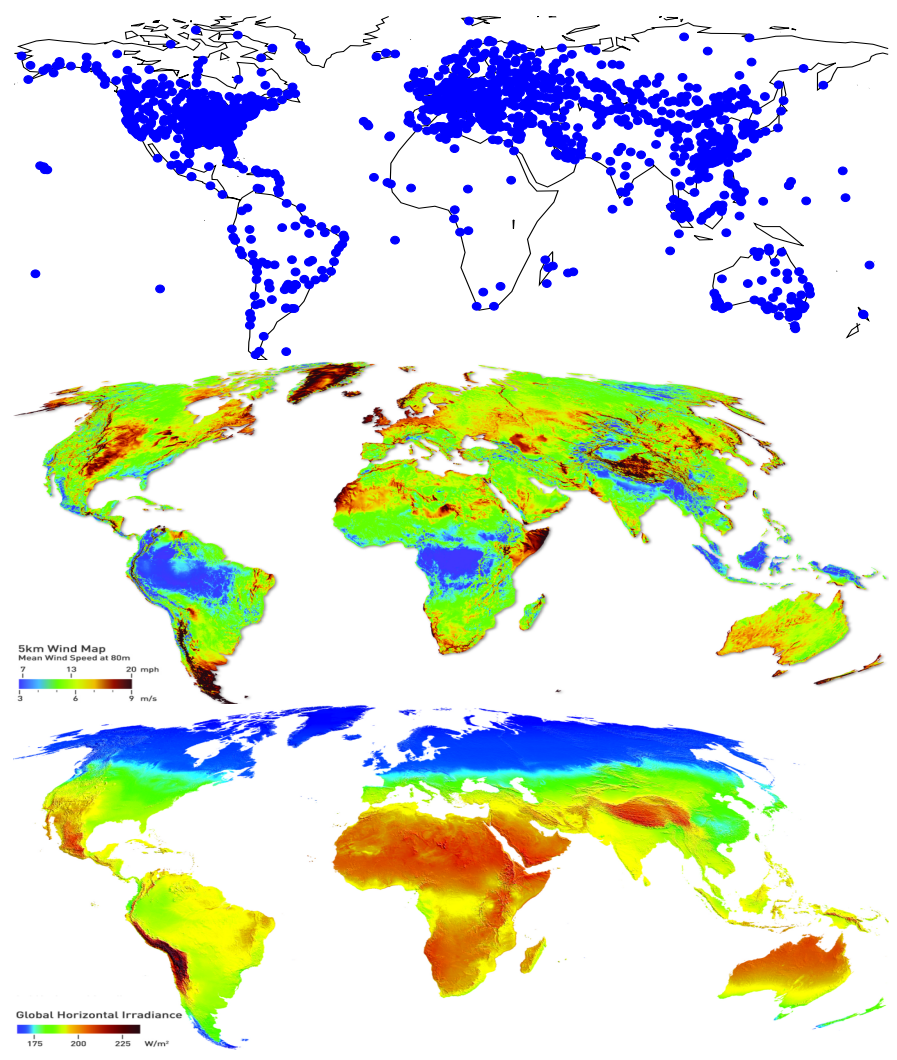

Figure 2. The top map plots our 1373 locations. The middle and bottom maps show the world-wide wind and solar availability [22], respectively.

specifications (including efficiency) for the 7.6MW E-126 wind turbine [24], TMY wind speeds and air pressures, and conversion losses. The wind capacity factor is the average of $\beta(d, t)$ over the year.

Fig. 3 shows the cumulative solar and wind capacity factors at the locations in our dataset. The figure shows a small number of locations where the wind capacity factor is much higher than that of solar. However, most of the locations have significantly higher solar capacity factors.

PUEs. Given the temperature at location $d$ during $t$, we compute $\operatorname{PUE}(d, t)$ using the function shown in Fig. 4. This function was generated using data collected from a micro-datacenter [18] equipped with a combination of an air-side economizer ("free cooling") and a direct-expansion air conditioner. We compute the average PUE for a location $d$ based on its $P U E(d, t)$ over the year. The average PUEs of our locations range from 1.06 to 1.13.

Fig. 5 plots the PUEs against wind and solar capacity factors. Interestingly, this figure shows that locations with the highest wind capacity factors typically have low PUEs, whereas locations with the highest solar capacity factors typically have high PUEs. However, there are many places with "reasonable" solar capacity factors (i.e., 15-23\%) that also have low PUEs (between 1.06 and 1.08).

Datacenter construction costs. The cost of building a datacenter (priceBuildDC(c)), including purchasing and 

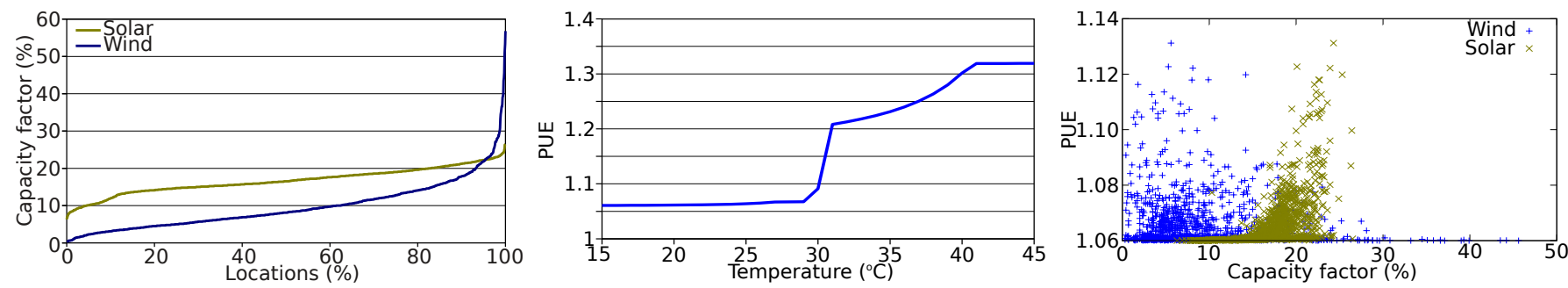

Figure 3. Capacity factors for our 1373 locations in Figure 4. PUE as a function of external temperature. increasing order.

Figure 5. PUE as a function of solar and wind capacity factors.

installing its cooling and power delivery infrastructures, typically depends on its maximum power $c$ [21]. Small datacenters $(\leq 10 \mathrm{MW})$ incur higher per Watt costs than large ones $(>10 \mathrm{MW})$. The specific cost per Watt depends on the target level of availability [21]. In all our studies below, we assume datacenters close to Tier III, costing \$15 (small) and $\$ 12$ (large) per Watt, with a typical availability of $99.827 \%$, as suggested by [25]. This cost is financed and amortized over 12 years, the expected lifetime of the datacenters.

Solar and wind costs. We set the installed costs of solar (priceBuildSolar) and wind (priceBuildWind) farms to $\$ 5.25$ [26] and \$2.1 [27] per Watt, respectively. This cost is financed over 12 years and amortized over 24 years, since solar panels and wind turbines have longer lifetimes (20-30 years) than the datacenter.

Land costs. We obtained the price of land (priceLand $(d)$ ) for locations in the US using the methodology presented in [28], which gets its data from a real-estate portal ${ }^{3}$ and calculates an average price for each location. We use data about industrial land with more than $1 \mathrm{~K}$ square-meters. For locations outside of the US, we obtained prices from multiple real-estate and financial Web sites and averaged the prices found for areas close to our locations. As the land cost is fully recoverable, the only incurred cost is that of financing, which we spread evenly over 12 years.

We instantiate the area required to build a DC (areaDC) as $0.56 \mathrm{~m}^{2}$ per $\mathrm{kW}$ [21], and the areas required for solar (areaSolar) and wind (areaWind) farms as 9.41 and 18.21 $m^{2}$ per $\mathrm{kW}$, respectively. The latter values were computed using information for existing wind ${ }^{4}$ and solar $^{5}$ farms. As a guideline, we chose the lowest values except for outliers that seemed completely inconsistent with the rest.

Power plants, transmission lines, and grid energy costs. We gathered data on power plants with capacities of $100 \mathrm{MW}$ or greater around the world from multiple Web sites. ${ }^{6}$ Then, given the distance between a datacenter and the nearest brown power plant, we use a cost of $\$ 310 \mathrm{~K}$ per $\mathrm{km}$ [29] for laying a power line to the datacenter (costLinePow). This cost is financed and amortized over 12 years.

\footnotetext{
${ }^{3}$ http://www.showcase.com

${ }^{4} \mathrm{http}: / /$ en.wikipedia.org/wiki/List_of_onshore_wind_farms

${ }^{5}$ http://en.wikipedia.org/wiki/List_of_photovoltaic_power_stations

${ }^{6}$ For example, http://en.wikipedia.org/wiki/List_of_power_stations
}

We instantiate the cost of grid energy (priceEnergy $(d)$ ) by gathering average prices per $\mathrm{kWh}$ from governmental and electricity user community portals. ${ }^{7}$ The average grid electricity price for our locations is around \$90/MWh.

With the growth of residential self-generation, the number of countries introducing some net metering scheme is increasing. However, there is still a wide range, with some countries not allowing net metering at all, and others that pay the full retail price for net metered energy. Looking to the future, our base evaluations assume the full retail price for all locations (i.e., credit NetMeter $=100 \%$ ). However, we also evaluate lower price points and their impact on the datacenter costs.

Battery efficiency and costs. We instantiate battery efficiency (battEff) as $75 \%$ and cost (priceBatt) as $\$ 200$ per $\mathrm{kWh}$ [30]. Batteries are replaced every 4 years [30], and this cost is financed and amortized over 4 years.

Network connection costs. We obtained a list of major IPv6 backbone connection points where we can directly connect datacenters. $^{8}$ Then, we use a cost of $\$ 300 \mathrm{~K}$ per km [29] to compute the cost of laying out fiber ( costLineNet $(d))$ from the nearest backbone connection point to each datacenter. This cost is financed and amortized over 12 years.

We assume that the amount of network bandwidth required by a datacenter grows linearly to the number of hosted servers. Our default amount is $1 \mathrm{Mbps}$ per server. A high bandwidth link to a backbone typically costs between $\$ 1$ and $\$ 2$ per Mbps. ${ }^{9}$ Our default cost is $\$ 1$ per Mbps.

Servers and internal networking costs. Our servers are Dell PowerEdge R610 with 4 2.66GHz cores and 6GBytes of RAM. They consume a maximum of $275 \mathrm{~W}$ and an average of $200 \mathrm{~W}$ at $30 \%$ utilization (a common average utilization in Internet service datacenters [25]). The cost of each server is around \$2000. Our interconnect is the 40-port Cisco Nexus 5020, which consumes 480W. Each switch connects to 32 servers and to each other hierarchically to reach all servers. The cost of each switch is $\$ 20 \mathrm{~K}$. All IT equipment is replaced every 4 years, and the costs are financed and amortized over 4 years.

\footnotetext{
${ }^{7}$ For example, http://www.eia.doe.gov for prices in US and http://www. energy.eu for prices in Europe.

${ }^{8}$ http://ipv6.nlsde.buaa.edu.cn

${ }^{9} \mathrm{http} / / / \mathrm{www}$. internetbandwidth.us
} 
TABLE II. SOME GOOD LOCATIONS FOR "BROWN", "SOLAR”, AND "WIND” that APPEAR IN DATACENTER Siting SOLUTIONS (SECTION IV).

\begin{tabular}{|c|c|c|c|c|c|c|c|c|c|}
\hline DC Type & Location & $\begin{array}{c}\text { DC Cost } \\
(\$ / \text { month }) \\
\end{array}$ & $\begin{array}{l}\text { Solar Cap. } \\
\text { Factor }(\%) \\
\end{array}$ & $\begin{array}{l}\text { Wind Cap. } \\
\text { Factor }(\%)\end{array}$ & $\begin{array}{l}\text { Max } \\
\text { PUE }\end{array}$ & $\begin{array}{c}\text { Electricity } \\
\text { (\$/MWh) }\end{array}$ & $\begin{array}{c}\text { Land } \\
\left(\$ / \mathbf{m}^{2}\right)\end{array}$ & $\begin{array}{c}\text { Distance } \\
\text { Power }(\mathbf{k m}) \\
\end{array}$ & $\begin{array}{c}\text { Distance } \\
\text { Network }(\mathbf{k m})\end{array}$ \\
\hline$\overline{\text { Brown }}$ & Kiev, Ukraine & 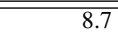 & & & 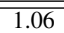 & 30 & 2200.0 & 22 & 7 \\
\hline \multirow{2}{*}{ Solar } & Harare, Zimbabwe & 16.5 & 22.4 & & 1.07 & 98 & 14.7 & 400 & 390 \\
\hline & Nairobi, Kenya & 13.1 & 20.9 & & 1.07 & 70 & 14.7 & 30 & 25 \\
\hline \multirow{2}{*}{ Wind } & Mount Washington, $\mathrm{NH}$, USA & 11.9 & & 55.6 & 1.06 & 126 & 947 & 345 & 71 \\
\hline & Burke Lakefront, OH, USA & 10.5 & & 20.9 & 1.06 & 58 & 329 & 409 & 3 \\
\hline
\end{tabular}

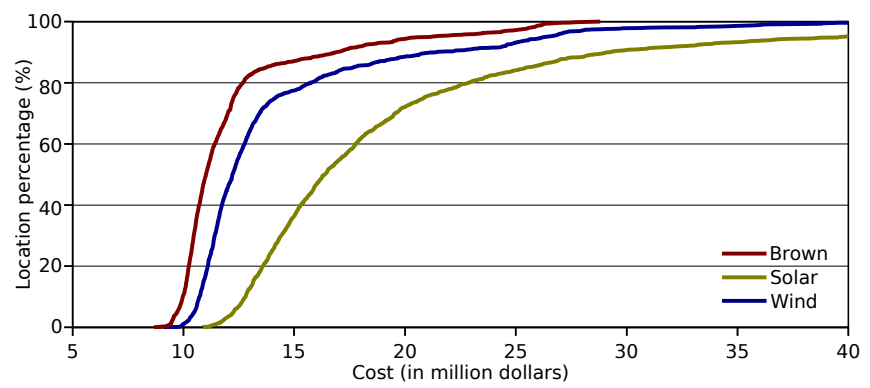

Figure 6. CDF of the per-month cost of building a $25 \mathrm{MW}$ datacenter with $50 \%$ green electricity at each of the 1373 locations.

Costs, amortization, and financing. Note that each CAPEX cost component has a specific amortization period. We also assume that each CAPEX cost is financed across a financing period of 12 years. We use an annual interest rate of $3.25 \% .^{10}$ In general, most values used to instantiate our framework parameters are from 2011 for consistency with [15].

\section{B. Total datacenter cost vs. location}

We now put together all the gathered data to explore costs vs. locations. Fig. 6 shows the distribution of the permonth cost of building a single datacenter providing 25MW of computing power at the 1373 locations. "Brown" is a datacenter with no green energy generation, while "Solar" and "Wind" have a solar and a wind farm, respectively, that generates $50 \%$ of the energy consumed by the datacenter over a year. These results assume that net metering is used to store excess green energy for later use. We study $25 \mathrm{MW}$ datacenters because this is a typical size in many solutions for a network of datacenters providing 50MW of computing power, a base case for our exploration of green cloud service siting (Section IV).

These results show that at $80 \%$ of the locations, a brown datacenter costs from $\$ 8.7 \mathrm{M}$ to $\$ 12.8 \mathrm{M}$ (per month), a datacenter with wind costs from $\$ 9.1 \mathrm{M}$ to $\$ 16 \mathrm{M}$, and a datacenter with solar costs from $\$ 10.9 \mathrm{M}$ to $\$ 23.3 \mathrm{M}$. Table II lists the attributes of some locations that appear in datacenter siting solutions in Section IV. Because of their high capacity factors, these are some of the cheapest locations for green energy production.

Interestingly, "Wind" is significantly cheaper than "Solar". This is because the price per $\mathrm{kW}$ for building a wind farm is less than half that of a solar farm (Table I). Further, net metering mitigates the higher variability of wind energy.

\footnotetext{
${ }^{10} \mathrm{http}: / /$ www.moneycafe.com/personal-finance/prime-rate
}

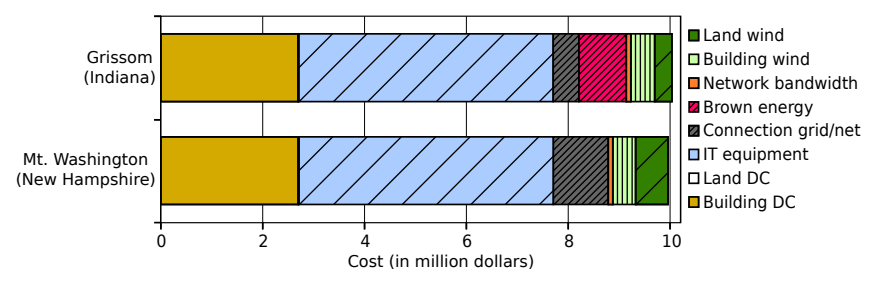

Figure 7. Costs of building a network of green datacenters with 50MW computation capacity and 50\% renewable energy.

Though more land is required for wind than solar, the land cost is fully recoverable (except for financing).

Note that our framework does not consider savings from governmental incentives for renewables, or costs associated with carbon taxes or cap-and-trade. Both of these factors would lower the cost of building green datacenters. Despite this pessimism, our results show that greener datacenters can be built at relatively low additional costs at good locations. This cost differential is even smaller when multiple locations are required for availability (Section IV). The cloud provider can accrue higher revenue from users who value green energy to compensate for this cost difference.

\section{Case study}

Finally, we demonstrate our tool by using it to site and provision a datacenter network providing 50MW of computation capacity, with at least $50 \%$ of the yearly energy consumption coming from on-site renewables. Fig. 7 shows the solution, including the locations and costs. This solution assumes that it is possible to use net metering at all locations.

The solution comprises two 25MW datacenters ( $\sim 91,000$ servers) in the US, costing $\$ 19.6 \mathrm{M} / \mathrm{month}$. One datacenter is placed in Mount Washington (Table II). A maximum PUE of 1.06 means that the total datacenter size is $26.5 \mathrm{MW}$. This location is fully powered by a $51 \mathrm{MW}$ wind farm and does not draw energy from the grid beyond what it net meters. Thus, no brown energy is consumed at this location. The second datacenter is placed in Indiana, where the maximum PUE is 1.07 and the wind capacity factor is $16.4 \%$. This datacenter has a small wind farm of $54 \mathrm{~kW}$ and draws an average of $26.5 \mathrm{MW}$ of brown power (almost all the total power) from the grid.

Note that the solution provisions exactly 50MW of computation capacity. This means that no computation resources are ever idled (due to load migration to another datacenter) because of insufficient green energy, even for a requirement of $50 \%$ green energy. At both locations, the main cost 


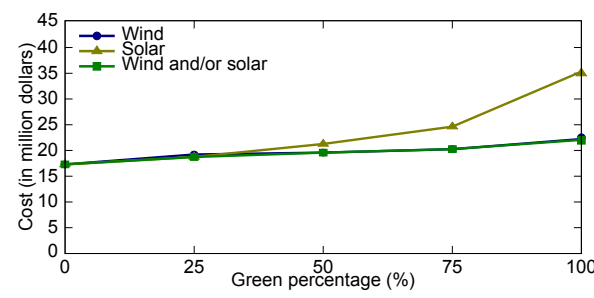

Figure 8 . Per-month cost with net metering vs. the desired percentage of green energy.

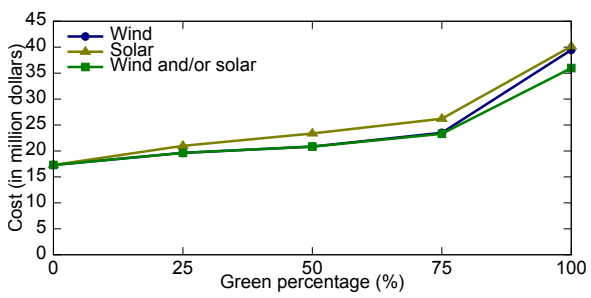

Figure 9. Per-month cost with battc

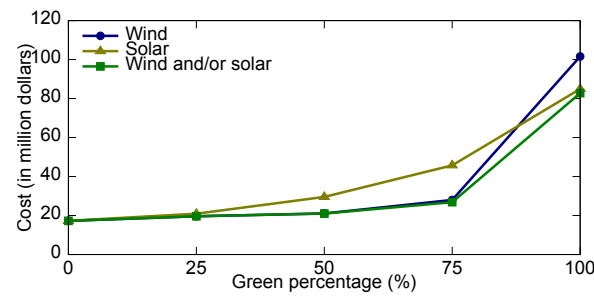

Figure 10. Per-month cost without storage vs. the desired percentage of green energy. components are the construction and IT equipment costs. In addition, locations in the US are often chosen because the US has areas with high capacity factors, cheap grid electricity, and extensive networking and power infrastructures.

\section{Execution time}

Using the heuristic solver described in Section II-C, our tool takes tens of minutes (e.g., $\sim 20$ minutes for a typical case) to produce a result when considering 50-100 potential locations. When considering the entire set of 1373 locations, the tool takes more than 2 weeks to produce reasonable results (the execution time grows exponentially with the number of locations). Solving the actual MILP formulation for 50-100 potential locations is possible (in one to several days of execution), when we have either a $0 \%$ or $100 \%$ green energy requirement. Our heuristic solver was able to find equally good solutions in all of these extreme cases.

\section{Green Datacenter Placement}

We now study the cost of building a green cloud service as a function of different parameters. Our technical report [31] also considers a stricter form of green energy enforcement.

\section{A. Desired percentage of green energy}

Fig. 8 shows the per-month cost of a network of datacenters providing $50 \mathrm{MW}$ of compute power, as a function of the desired percentage of green energy. Here, we assume that net metering is used for green energy storage. The "Wind and/or solar" (or simply solar+wind) curve represents the solution in which we allow either or both technologies to be used.

The leftmost point in the figure represents the $0 \%$ desired green energy, i.e. the cheapest "Brown" network out of our world-wide locations. Thus, the figure shows that it is possible to build a green datacenter network at relatively low cost. For example, a datacenter network with $50 \%$ green energy $(\$ 19.6 \mathrm{M} / \mathrm{month})$ is only about $13 \%$ more expensive than the cheapest network with no green energy ( $\$ 17.3 \mathrm{M} / \mathrm{month})$. We detailed the $50 \%$ green energy solution in Section III-C.

In comparison, a solar-only green datacenter network is more expensive $(\$ 21.3 \mathrm{M} /$ month) for a $50 \%$ green energy requirement. The main reasons are: (1) solar's higher cost per installed Watt; (2) the higher capacity factors of the best wind locations; and (3) the use of net metering to store surplus green energy, which mitigates the high variability of wind. Thus, the best solution builds two wind plants totaling $51 \mathrm{MW}$ of peak production. In contrast, the solaronly solution requires three datacenters located in Kiev, Nairobi, and Iowa, with solar plants totaling 168.5MW.

When we desire $100 \%$ green energy ( $\$ 22.1 \mathrm{M} / \mathrm{month})$, the network is about $28 \%$ more expensive than a network with no green energy. In this case, the best solution comprises two datacenters located in Mount Washington and Burke Lakefront (Table II), with wind plants totaling 222.2MW of peak production, and a solar plant at Burke Lakefront providing $32.9 \mathrm{MW}$ of peak production. Interestingly, using a combination of solar and wind, the solution still provisions exactly 50MW of computation power. Though it is difficult to see this in the figure, the solar+wind solution is only slight cheaper than wind-only at $100 \%$ green energy.

\section{B. Net metering return}

In the results above, we assume that net metering has a return of $100 \%$, i.e. the revenue accrued by net metering $1 \mathrm{kWh}$ of energy is the same as the price of drawing $1 \mathrm{kWh}$ of grid electricity. In other words, storage of green energy in the grid is free. However, power utilities may pay less than the full retail price for each $\mathrm{kWh}$ stored in the grid.

Interestingly, we find that the net metering return does not have a significant impact on the total network cost. For example, the wind-only and solar+wind datacenter networks cost roughly $\$ 22 \mathrm{M} /$ month with $100 \%$ green energy, regardless of the net metering return. However, the ability to store green energy is critical when we desire large percentages of green energy, even when the power utility does not pay for the net metered energy. For example, when storage is not available, the wind-only cost increases by almost $\$ 80 \mathrm{M} /$ month with $100 \%$ green energy, due to the high variability of wind energy. We discuss storage further next.

\section{Green energy storage}

Net metering is not available in many parts of the world. Fig. 9 shows the costs when we use batteries to store green energy, instead of net metering. Generally, the costs follow the trends shown for net metering, although storage with batteries is more expensive. As large battery capacity is expensive, the green power plants have to be larger to compensate. At $100 \%$ green energy requirement, wind-only is almost as expensive as solar-only. The reason is that a large amount of batteries has to be provisioned to deal with 


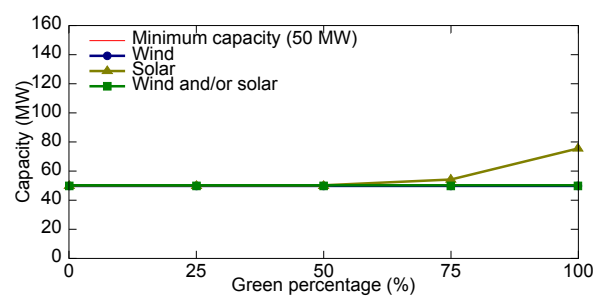

Figure 11. Total compute capacity with net metering vs. the desired percentage of green energy.

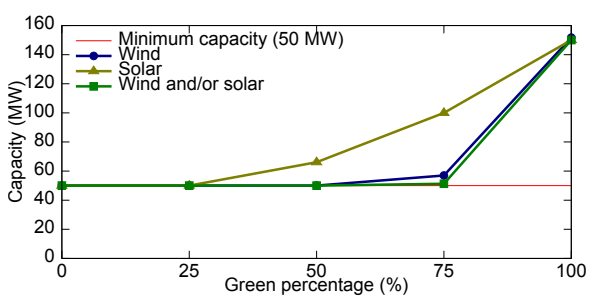

Figure 12. Total compute capacity without storage vs. the desired percentage of green energy.

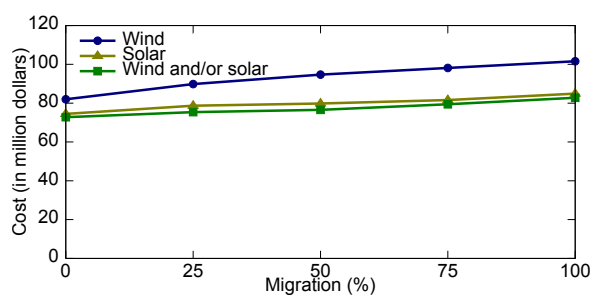

Figure 13. Per-month cost of a $100 \%$ green datacenter network without storage, as a function of the migration requirements. the high wind variability. The solar+wind solution uses both solar and wind to offset variability in each of the sources, and thus requires only about half as much battery capacity.

Although one can use batteries to replace net metering, a serious problem is that the most popular technology in datacenter batteries (lead-acid) uses materials that are harmful to the environment. Thus, we now consider scenarios in which no energy storage is available. Fig. 10 plots the cost in this case. As mentioned above, the cost increases significantly when a high percentage of green energy is desired and storage is unavailable. For example, the cheapest $100 \%$ green datacenter network is now $3.75 \mathrm{x}$ more expensive than when net metering is available ( $\$ 82.8$ vs. $\$ 22.1 \mathrm{M} / \mathrm{month})$. The reason is that more datacenters are needed to ensure that green energy is always available, e.g. the $100 \%$ wind-only solution needs 13 datacenters. Also, the green energy plants need to be larger, to ensure that there is always sufficient green energy being produced. The solution found for $100 \%$ green energy with solar and/or wind includes 3 datacenters totaling $150 \mathrm{MW}$ of compute power, $1.1 \mathrm{GW}$ of solar power, and $38 \mathrm{GW}$ of wind power.

Interestingly, wind-only becomes the most expensive at $100 \%$. This is because wind energy is so variable that it requires massive over-provisioning to ensure $100 \%$ green energy when no storage is available. On the other hand, cost rises more rapidly for solar-only solutions at lower green energy requirements, because substantially less solar energy is produced early in the day and later in the afternoon. Thus, to avoid building more datacenters, solar-only solutions over-provision the solar plants to supply sufficient energy during the periods of low production. All load is migrated away from a datacenter at night time, when no solar energy is produced. This is why solar-only solutions site three datacenters roughly evenly spread around the world in the absence of storage.

\section{Datacenter idleness when following renewables}

There is often a perception that following the renewables would leave a significant amount of idle compute capacity in datacenters. Figs. 11 and 12 plot the total provisioned compute capacity, as a function of the desired percentage of green energy. Observe that except for the cases of $100 \%$ solar energy, $100 \%$ green energy without storage, and $\geq 50 \%$ solar-only energy and no storage, there is very little idleness in the green datacenter networks. This shows that networks of green datacenters can be built without significant overprovisioning of capacity for following the renewables, except at very high percentages of required green energy.

\section{E. Migration impact}

The results above assume that, whenever load needs to be migrated from one datacenter to another, the migration requires a full epoch (i.e., 1 hour). This is conservative, since we may be moving only a small amount of computation.

Fig. 13 studies the impact of the migration time (its associated energy consumption) on the total cost. In particular, the figure plots the cost of a $100 \%$ green datacenter network with no storage vs. migration time of $0 \%$ to $100 \%$ of an epoch. We only show results for this case because, at a lower percentage of green energy and/or when there is storage, there are very few migrations and so migration overheads are insignificant.

These results show that smaller migration energy overheads can reduce the cost of the best solution up to $12 \%$. The reduction for wind-only is even greater (19\%), because of its large number of migrations across the 13 locations.

\section{F. Summary}

We made several interesting observations from this study:

- With net metering, we can build a green cloud service at a low additional cost compared to the best possible brown service, e.g. $13 \%$ when we desire $50 \%$ green energy use.

- In most cases, wind is more cost-effective than solar for powering a cloud service for two main reasons: wind is cheaper per $\mathrm{kW}$ of installed capacity, and energy storage mitigates the greater variability of wind energy.

- There is only one scenario in which solar is more costeffective than wind: when there is no storage, and we want a high percentage of green energy. The reason is that solar energy is less variable and, thus, more predictable.

- Despite the variability in renewable energy, we only need a few datacenters (typically 2-3) in the presence of storage, even when we desire a high percentage of green energy. 


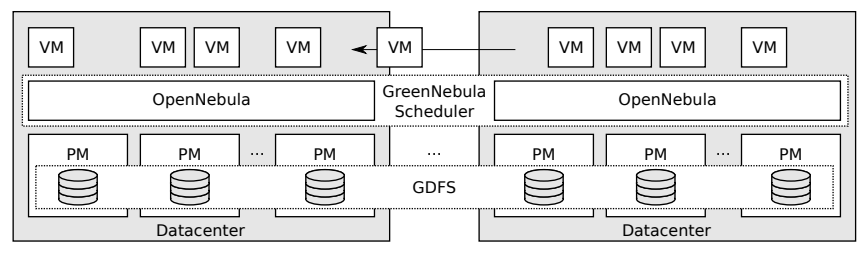

Figure 14. The GreenNebula architecture.

- Energy storage decreases the total cost by over $60 \%$, when we desire a $100 \%$ green service. In particular, net metering reduces the total cost by more than $73 \%$.

- The main benefit of net metering is its capacity to store green energy and not the actual net metering revenue. In fact, the net metering revenue has little impact on the service's cost.

- Follow the renewables rarely requires non-trivial extra compute capacity, when wind energy is used or there is storage.

- With enough storage, migration energy overheads for following renewables are minimal. Otherwise, they can increase the cost by at least $15 \%$.

\section{GReenNebula}

We now describe GreenNebula, a prototype VM placement system that we have built to demonstrate the feasibility of a follow-the-renewables HPC cloud service.

\section{A. Design and implementation}

Fig. 14 shows the GreenNebula architecture. GreenNebula is built around the OpenNebula within-datacenter virtualization platform [13], and comprises two components: a multidatacenter scheduler, and a multi-datacenter distributed file system. The scheduler runs on one of the datacenters (it can be replicated for high availability). Every hour, the scheduler predicts the green energy production at each datacenter 48 hours into the future [15], [17], [32], communicates with the other datacenters to collect current workload information (i.e., average power usage), computes how the workload should be re-partitioned across the datacenters in the next time window, and instructs each datacenter about any migrations it should perform. The workload partitioning is a variant of the optimization problem presented in Section II, where we fix the locations and provisioning of the datacenters, and remove the constraint for a minimum percentage of green energy. This optimization then becomes a small MILP problem (that can be solved efficiently with existing solvers) seeking to minimize the brown energy used over the next 48 hours, while considering both the predictions of green energy production and the energy overhead of migration. The performance overhead of migration on the VMs is typically negligible, as GreenNebula uses live migration; i.e., the applications running on the VMs keep running normally during migration.

Once the scheduler determines the fraction of load that should be placed at each datacenter, it decides the migration
TABLE III. NETWORK FOR 100\% GREEN ENERGY WITHOUT STORAGE.

\begin{tabular}{l|rrr} 
Location & IT Capacity & Solar & Wind \\
\hline Mexico City, Mexico & $50.0 \mathrm{MW}$ & $327.7 \mathrm{MW}$ & $9.0 \mathrm{~kW}$ \\
Andersen, Guam & $50.0 \mathrm{MW}$ & $375.4 \mathrm{MW}$ & $38.0 \mathrm{MW}$ \\
Harare, Kenya & $50.0 \mathrm{MW}$ & $396.7 \mathrm{MW}$ & $20.8 \mathrm{~kW}$
\end{tabular}

schedule. Specifically, it orders the datacenters in decreasing amount of load (i.e., power) to be migrated out. It then uses a first fit strategy to migrate VMs from each "donor" to the closest "receiver". After being informed of the schedule, the donor datacenters effect the migrations, choosing VMs with smaller memory/disk footprints before larger ones, until the desired amount of power has been migrated out.

The design of GreenNebula's file system (GDFS) is similar to that of HDFS [33] (i.e., one master holding name bindings and meta-data, multiple workers storing replicas of data blocks), but allows for file mutation. Data blocks are replicated across the datacenters. When a write occurs, GDFS writes the local replica, and invalidates the remote replicas (by updating the meta-data at the master). If there is no valid local replica and the write is not for an entire block, GDFS first obtains a copy from another datacenter. Written blocks are re-replicated in the background. This design keeps the response time for writes low, yet allows VMs to be easily migrated between datacenters; only the recently modified blocks that have not been replicated need to be moved with a migrating VM.

\section{B. Validation experiments}

As we do not have access to multiple datacenters or commercial cloud services do not provide live migration, we experiment with GreenNebula in emulation mode. Specifically, we use three servers to emulate three datacenters. We then load the servers with VMs running inside Oracle's VirtualBox [34]. The workload comprises 9 equally configured VMs. Each VM has one virtual CPU, 512MB of memory, and a 5GB disk; runs a CPU-intensive synthetic application that also generates $110 \mathrm{MB}$ of disk data per hour; and consumes $30 \mathrm{~W}$.

A real cloud service would have high guaranteed network bandwidth between its datacenters, perhaps via leased links. As we do not have access to such networking, we collected migration bandwidth data using regular Internet measurements between Barcelona, Spain and Piscataway, NJ. Specifically, using a VPN, GreenNebula is able to migrate VMs with a memory footprint and disk data changes totaling over $750 \mathrm{MB}$ in less than one hour. Recall that how long a migration takes is irrelevant in terms of the performance of the applications running on the VM, since GreenNebula uses live migration.

For simplicity, we assume perfectly accurate predictions of green energy production. Previous works have shown that this production can be predicted with high accuracy [15], [17], [32]. 


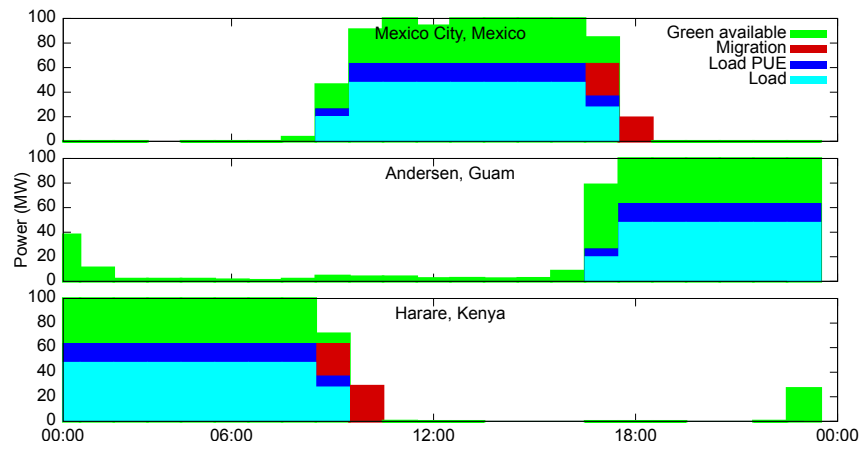

Figure 15 . Load distribution to achieve $100 \%$ green energy without energy storage. The green area shows the amount of green energy produced, light blue shows the computing load, dark blue shows the PUE energy overheads, and red shows energy used by migrations.

\section{Follow-the-renewables experiments}

When green energy storage is available, GreenNebula does not need to migrate much load. Thus, we now consider its behavior in the absence of storage. Table III describes the datacenters required to use $100 \%$ green energy with no storage, again assuming 50MW of required compute power. In this placement, the three datacenters are powered by a mix of solar and wind. As we have seen in Section III, this setup uses (mostly) solar energy as it is more predictable.

Fig. 15 shows a clear follow-the-renewables pattern during a day of the TMY data. GreenNebula starts hosting the load in Kenya, then it moves the load to Mexico, and finally to Guam. The figure clearly shows that green energy capacity is overbuilt to support days with low green energy availability. The figure also shows the relatively small energy overhead of migrating load between locations.

GreenNebula computes the workload distribution efficiently. For the example of Fig. 15, it computes each migration schedule in just $240 \mathrm{~ms}$ on average, on a $2 \mathrm{GHz}$ Intel machine. For the same requirements but using solaronly and wind-only plants, the average times are 240 and $310 \mathrm{~ms}$, respectively.

The computation times scale nicely for larger problems. For example, assuming a requirement of 200MW of IT compute power, GreenNebula computes each migration schedule in 760, 780, and $760 \mathrm{~ms}$ on average for solar-only, wind-only, and solar+wind setups, respectively. When net metering is available, it computes the schedule even faster, requiring $160 \mathrm{~ms}$ on average for all three setups.

\section{RELATED WORK}

Datacenter site selection. To the best of our knowledge, no prior works have considered the siting and provisioning of datacenters (at least partially) powered by on-site solar and wind resources for a cloud service. The two closest related works are [19] and [11]. Goiri et al. [19] focused on siting and provisioning datacenters for interactive Internet services in the US. Unlike our work, they did not consider follow-therenewables cloud services, selecting sites for building new renewable energy plants, solar or wind energy characteristics (i.e., costs, efficiencies, capacity factors, variability), different percentages of green energy, or green energy storage.

Recently, Gao et al. [11] studied siting datacenters near existing wind farms, and distributing load using a greedy online algorithm. However, they did not consider selecting sites for building new renewable plants, solar energy, energy storage, datacenter costs, or renewable plant costs.

Researchers have also considered the software layer and its topology when siting datacenter networks [35].

Following the renewables across datacenters. Le et al. [12] first studied following renewables in green datacenter networks. Stewart and Shen [36] discussed the topic, but did not include a system or an evaluation. More recent studies have considered load distribution algorithms in follow-the-renewables services [7]-[11].

The prior works did not implement real follow-therenewables workload managers, relying on simulations and modeling. The exception is [7], which presented a prototype (interactive) request distribution system for a multidatacenter Internet service. In contrast, we design and implement a real (batch) VM placement/migration system (on top of a widely used VM manager) for a multi-datacenter cloud service.

Other works have studied load distribution across datacenters [37]-[39] or real (and sometimes live) VM migrations across datacenters [40], [41]. Unfortunately, neither group of works considered renewables.

Rahman et al. [42] present an excellent survey of prior works on geographic load balancing.

\section{CONCLUSIONS}

In this paper, we studied the problem of building an HPC cloud service comprising datacenters that are powered at least partially by on-site renewable sources of energy. In this context, we first proposed a cost-driven framework, optimization problem, and solution approach for siting and provisioning green datacenters for a follow-the-renewables HPC cloud service. Next, we introduced GreenNebula, a real follow-the-renewables VM placement and migration system that extends OpenNebula, a widely used system for withindatacenter VM management.

Our optimization results prompted several interesting observations, including the scenarios under which using wind energy is cheaper than solar energy (and vice-versa), the cost implications of green energy storage, the impact of different desired percentages of green energy, and the impact of the energy overhead of VM migration. Our GreenNebula experience and results illustrated that it is possible to build followthe-renewables HPC cloud services with low overhead.

\section{ACKNOWLEDGMENT}

This work has been partially supported by NSF grant CSR-1117368, the Rutgers Green Computing Initiative, and 
the BSC-CNS Severo Ochoa program and the TIN201234557 project with funding from the European Commission's 7th Framework Program under contract 608679 (RenewIT) and the Spanish Ministry of Economy and Competitivity and the European Unions FEDER funds.

\section{REFERENCES}

[1] Apple, "Apple and the Environment," 2013, http://www.apple.com/environment/renewable-energy/.

[2] R. Miller, "Data Centers Scale Up Their Solar Power," 2012.

[3] AISO.net, "Web Hosting as Nature Intended," 2012, http:// www.aiso.net/.

[4] Green House Data, "An Economically Responsible Data Center," 2012, http://www.greenhousedata.com/.

[5] GreenQloud, http://greenqloud.com.

[6] UK Government, "CRC Energy Efficiency Scheme," 2013, https://www.gov.uk/crc-energy-efficiency-scheme/.

[7] K. Le et al., "Capping the Brown Energy Consumption of Internet Services at Low Cost," in IGCC, 2010.

[8] M. Lin et al., "Online algorithms for geographical load balancing," in $I G C C, 2012$.

[9] Z. Liu et al., "Geographical load balancing with renewables," in GreenMetrics, 2012.

[10] Y. Zhang, Y. Wang, and X. Wang, "GreenWare: Greening Cloud-Scale Data Centers to Maximize the Use of Renewable Energy," in Middleware, 2011.

[11] Y. Gao et al., "The Answer Is Blowing in the Wind: Analysis of Powering Internet Data Centers with Wind Energy," in INFOCOM, 2013.

[12] K. Le et al., "Cost- And Energy-Aware Load Distribution Across Data Centers," in HotPower, 2009.

[13] D. Milojicic, I. M. Llorente, and R. S. Montero, "OpenNebula: A Cloud Management Tool," IEEE Internet Computing, vol. 15, 2011.

[14] EcobusinessLinks, "Green Hosting - Sustainable Solar \& Wind Energy Web Hosting," 2012, http://www.ecobusinesslinks.com/green_webhosts/.

[15] I. Goiri et al., "GreenSlot: Scheduling Energy Consumption in Green Datacenters," in SC, November 2011.

[16] C. Ren et al., "Carbon-Aware Energy Capacity Planning for Datacenters," in MASCOTS, 2012.

[17] I. Goiri et al., "GreenHadoop: Leveraging Green Energy in Data-Processing Frameworks," in EuroSys, 2012.

[18] _ _ "Parasol and GreenSwitch: Managing Datacenters Powered by Renewable Energy," in ASPLOS, 2013.

[19] — , "Intelligent Placement of Datacenters for Internet Services," in ICDCS, 2011.

[20] D. Siewiorek and R. Swarz, Reliable Computer Systems Design and Evaluation. A. K. Peters, 1998.

[21] W. P. Turner and J. H. Seader, "Dollars per KW Plus Dollars per Square Foot are a Better Data Center Cost Model than Dollars per Square Foot Alone," in Uptime Institute, 2006.
[22] 3TIER, "Resource maps," http://www.3tier.com/en/support/ resource-maps/.

[23] T. Huld et al., "Mapping the Performance of PV Modules, Effects of Module Type and Data Averaging," Solar Energy, vol. 84, no. 2, 2010.

[24] Enercon, "Enercon Product Overview," 2012, http://www. enercon.de/p/downloads/EN_PUE_en_web.pdf.

[25] L. A. Barroso and U. Hölzle, The Datacenter as a Computer: An Introduction to the Design of Warehouse-Scale Machines, 2009.

[26] G. Barbose, N. Darghouth, and R. Wiser, "Tracking the Sun V,” LBNL, Tech. Rep. LBNL-5919E, 2012.

[27] R. Wiser and M. Bolinger, "2011 Wind Technologies Market Report," U.S. Department of Energy, Tech. Rep., 2012.

[28] J. B. Nichols, S. D. Oliner, and M. R. Mulhall, "Commercial and Residential Land Prices Across the United States," Finance and Economics Discussion Series, 2010.

[29] T. Seppa, "Improving Asset Utilization of Transmission Lines by Real Time Rating," 1999.

[30] D. Wang et al., "Energy Storage in Datacenters: What, Where, and How Much?" in SIGMETRICS, 2012.

[31] J. L. Berral et al., "Cost-Aware Building of Cloud Services Powered by Renewable Energy," Rutgers University, Tech. Rep., 2013.

[32] B. Aksanli et al., "Utilizing Green Energy Prediction to Schedule Mixed Batch and Service Jobs in Data Centers," in HotPower, 2011.

[33] D. Borthakur, "HDFS Architecture Guide," 2008, http:// hadoop.apache.org/common/docs/current/hdfs_design.pdf.

[34] Oracle, "VirtualBox," 2008, http://www.virtualbox.org.

[35] F. Larumbe and B. Sansò, "Optimal Location of Data Centers and Software Components in Cloud Computing Network Design," in CCGrid, 2012

[36] C. Stewart and K. Shen, "Some Joules Are More Precious Than Others: Managing Renewable Energy in the Datacenter," in HotPower, 2009.

[37] K. Le et al., "Reducing Electricity Cost Through Virtual Machine Placement in High Performance Computing Clouds," in SC, 2011.

[38] L. Rao et al., "Minimizing Electricity Cost: Optimization of Distributed Internet Data Centers in a Multi-ElectricityMarket Environment," in INFOCOM, 2010.

[39] R. Wang et al., "Data Centers as Controllable Load Resources in the Electricity Market," in ICDCS, 2013.

[40] T. Hirofuchi et al., "A Live Storage Migration Mechanism Over WAN for Relocatable Virtual Machine Services on Clouds," in CCGridd, 2009.

[41] T. Wood et al., "CloudNet: Dynamic Pooling of Cloud Resources by Live WAN Migration of Virtual Machines," in VEE, 2011.

[42] A. Rahman, X. Liu, and F. Kong, "A Survey on Geographic Load Balancing Based Data Center Power Management in the Smart Grid Environment," IEEE Communications Surveys and Tutorials, vol. 16, no. 1, 2014. 